\title{
A theoretical basis for practical amplifier design using cascaded operational amplifiers
}

David J. Comer

comer.ee@byu.edu

Follow this and additional works at: https://scholarsarchive.byu.edu/facpub

Part of the Electrical and Computer Engineering Commons

\section{Original Publication Citation}

Comer, D. J. "A Theoretical Basis for Practical Amplifier Design using Cascaded Operational

Amplifiers." Electronic Circuits and Systems, IEE Proceedings G 132.4 (1985): 161-4

\section{BYU ScholarsArchive Citation}

Comer, David J., "A theoretical basis for practical amplifier design using cascaded operational amplifiers" (1985). Faculty Publications. 754.

https://scholarsarchive.byu.edu/facpub/754

This Peer-Reviewed Article is brought to you for free and open access by BYU ScholarsArchive. It has been accepted for inclusion in Faculty Publications by an authorized administrator of BYU ScholarsArchive. For more information, please contact ellen_amatangelo@byu.edu. 


\title{
A theoretical basis for practical amplifier design using cascaded operational amplifiers
}

\author{
Prof. D.J. Comer, B.S., M.S., Ph.D., Sen.Mem.I.E.E.E. \\ Indexing term: Amplifiers \\ Abstract: A procedure is developed for the design of amplifiers to meet specified gain and bandwidth figures. \\ The number of required operational amplifier stages is minimised. A figure of merit is introduced to determine if \\ a particular operational amplifier can be used to satisfy a given set of specifications.
}

1

Introduction

Most electronics textbooks treat single stage operational amplifier designs $[1,2]$, but many practical amplifiers require multiple, cascaded operational amplifier stages to meet the given specifications. Two of the most significant specifications of amplifier performance are the overall voltage gain and the overall upper $3 \mathrm{~dB}$ frequency or bandwidth. In order that a finished design be considered acceptable, the actual overall gain and bandwidth must equal or exceed the specified values. An additional constraint imposed in practice is that of minimising the component cost of the finished product. Two dominant factors in determining overall component cost are the cost of the operational amplifier and the number of stages required.

A practical design procedure should allow the designer to [3]

(a) easily determine if a given operational amplifier can be used to satisfy overall gain and bandwidth specifications

(b) minimise the number of stages required for the design

(c) determine the individual stage gains and bandwidths required to meet the overall specifications.

(a) and (b) must be jointly considered to minimise component cost. A very expensive operational amplifier may lead to fewer stages required, yet total cost may be decreased by using more stages of a cheaper operational amplifier.

Although a good deal of amplifier theory has been developed in past years, it has been directed towards the goal of achieving a required overall gain along with maximum possible bandwidth [4-6]. When the resulting bandwidth exceeds the specified value, it is obvious that the number of stages could be reduced. In the past, the only method used to reduce stages was trial and error. This is by no means a trivial task. When the number of stages is reduced from the number used to maximise overall bandwidth, the individual stage gains must be adjusted; the individual stage bandwidths will consequently change, and the factor relating overall bandwidth to individual stage bandwidth also changes. Use of trial-anderror methods is highly inefficient. The theory and procedure developed in this paper allows the designer to meet specifications without resorting to a trial and error approach. Appropriate operational amplifiers can be selected and cost can be minimised with this procedure.

Paper 3916G (E10), first received 26th September 1984 and in revised form 11th January 1985

The author is with the Electrical Engineering Department, Brigham Young University, 459 CB, Provo, Utah 84602 , USA

\section{The operational amplifier}

The basic element in this procedure is the operational amplifier compensated for unity gain, shown in Fig. 1.
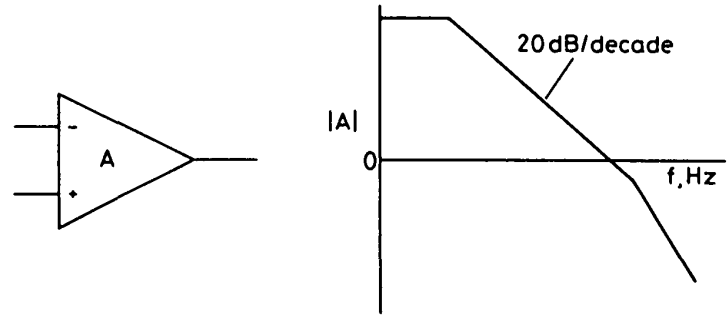

Fig. 1 The operational amplifier and frequency response

These devices exhibit a $20 \mathrm{~dB} /$ decade roll off in gain above the upper corner frequency and allow gain and bandwidth to be exchanged directly [7]. The gain-bandwidth product $(G B W)$ is then constant with gain changes. The direct exchange of gain and bandwidth is possible for gains equal to or greater than unity. by

For the noninverting stage of Fig. 2(a), the gain is given

$$
A_{s}=1+R_{F} / R_{2}
$$

whereas the inverting stage of Fig. $2(b)$ has a gain of

$$
A_{s}=-R_{F} / R_{1}
$$
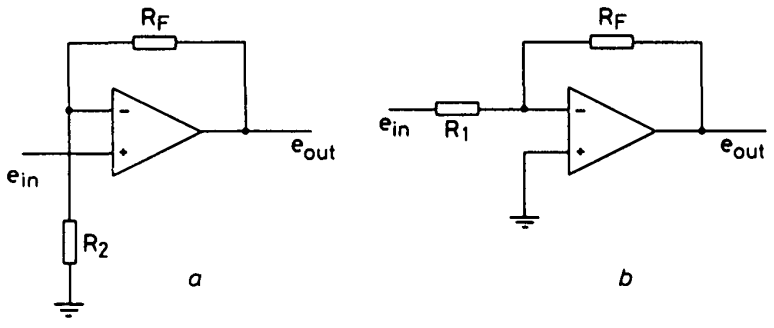

D

Fig. 2 Operational amplifier stages

$a$ Noninverting

$b$ Inverting

The bandwidth in either case can be found from

$$
f_{s}=G B W / A_{s}
$$

Not only do most commercial operational amplifiers have a constant $G B W$, the high input and low output impedances of these devices lead to negligible interaction of stages when cascaded. Thus, gain and bandwidth of an individual stage can be adjusted without affecting gain or bandwidth of adjacent stages. 
Most amplifier designs specify a value of required gain and required bandwidth that cannot be achieved by a single operational amplifier stage. When more than one stage is used, the overall gain and bandwidth values become rather complex functions of the individual stage values. If $n$ identical or iterative stages are used, the overall gain $A_{0}$ is expressed as

$$
A_{0}=A_{s}^{n}
$$

where $A_{s}$ is the individual stage gain. The overall bandwidth $f_{0}$ is given in terms of the individual stage bandwidth $f_{s}$ as

$$
f_{0}=f_{s}\left(2^{1 / n}-1\right)^{1 / 2}
$$

Eqn. 2 is referred to as the bandwidth shrinkage equation [5]. For $n>2$, eqn. 2 can be approximated quite accurately [5] by

$$
f_{0}=f_{s} / 1.2 n^{1 / 2}
$$

If constant $G B W$ stages are used, the overall bandwidth can be maximised with respect to individual stage gain. This is accomplished by expressing $f_{0}$ in terms of $A_{s}$ to give

$$
f_{0}=G B W\left(\ln A_{s}\right)^{1 / 2} / 1.2 A_{s}\left(\ln A_{0}\right)^{1 / 2}
$$

Eqn. 4 is then differentiated with respect to $A_{s}$ and this derivative is equated to zero. The result of this maximisation process is well-known $[4,5]$ and states that the individual stage gain must equal 1.65 to yield the maximum overall bandwidth with the given $G B W$.

This theory can be used to calculate the minimum required $G B W$ of an operational amplifier for use in an amplifier. If $A_{\text {or }}$ and $f_{\text {or }}$, the required overall gain and bandwidth, respectively, are specified, the gain can be taken to be 1.65 . The actual overall bandwidth from eqn. 4 must exceed the required bandwidth or

$$
G B W\left(\ln A_{s}\right)^{1 / 2} / 1.2 A_{s}\left(\ln A_{o r}\right)^{1 / 2} \geqslant f_{o r}
$$

assuming the overall gain is caused to equal $A_{o r}$. Substituting 1.65 for $A_{s}$ and solving for $G B W$ gives

$$
G B W \geqslant 2.8 f_{o r}\left(\ln A_{o r}\right)^{1 / 2}
$$

If the operational amplifier $G B W$ does not equal or exceed the value given in eqn. 5 , the amplifier specifications cannot be met regardless of the number of such stages used. If the operational amplifier $G B W$ happens to equal the minimum value, the amplifier can be realised only if the individual stage gain is set to 1.65 . The number of stages required in this instance is

$$
n=\ln A_{\text {or }} / \ln 1.65 \simeq 2 \ln A_{\text {or }}
$$

From a practical standpoint the most interesting case occurs when the operational amplifier $G B W$ exceeds the minimum value. The amplifier could now be constructed to equal or exceed both the overall gain and bandwidth requirements. Rather than allow excess gain or bandwidth, however, it is economically advantageous to reduce the number of stages to the minimum required to achieve the overall specifications. In many cases, this results in a significant reduction in the number of stages and component cost of the amplifier.

Before proceeding to the calculation of the minimum number of stages required, another result must be established. This result applies to noniterative stages as well as iterative. Given any number of stages and a required overall bandwidth, it has been shown that gain is maximised by setting individual stage bandwidths equal [8].
Using this result allows the bandwidth shrinkage formula to apply to noninterative amplifiers in which gain is being maximised.

\section{$4 \quad$ Minimising the number of stages}

To calculate the minimum number of operational amplifier stages needed to satisfy a given design, it is first assumed that $n$ stages with an individual stage gain of 1.65 are used. Assuming an excess overall bandwidth results from this situation, stages can be removed if the proper adjustment is made in individual stage gain and bandwidth. If $m$ is the minimum number of stages, the adjusted single stage gain must be

$$
A_{s}=(1.65)^{n / m}=\left(A_{o r}\right)^{1 / m}
$$

The adjusted single stage bandwidth can be found from eqn. 3 to be

$$
f_{s}=1.2 m^{1 / 2} f_{\text {or }}
$$

To solve for the three unknowns $A_{s}, f_{s}$ and $m$, a third equation is required. The simple relationship between gain and bandwidth yields this third equation, that is,

$$
G B W=A_{s} f_{s}
$$

Solving for $m$ from the two preceding equations results in

$$
m=G B W^{2} / 1.44 f_{o r}^{2} A_{s}^{2}
$$

Taking the natural logarithm of both sides of eqn. 7 gives

$$
\ln A_{s}=\left(\ln A_{o r}\right) / m
$$

Eliminating $m$ from the last two equations leads to the requirement on $A_{s}$ that

$$
\ln A_{s}=Z A_{s}^{2}
$$

where

$$
Z=1.44 f_{o r}^{2}\left(\ln A_{o r}\right) / G B W^{2}
$$

For a given value of $Z$, eqn. 8 can be solved graphically or with the aid of a hand calculator. Table 1 tabulates values of $A_{s}$ for various practical values of $Z$. The range of individual stage gain from 1.65 to 10 is sufficient for most practical amplifier stages. Larger values of gain limit the individual stage bandwidth to impractical values.

Once the proper value of $A_{s}$ is determined, the minimum number of stages can be calculated from eqn. 7 and is

$$
m=\ln A_{o r} / \ln A_{s}
$$

Table 1 : Individual stage gain as a function of $Z$

\begin{tabular}{llll}
\hline$Z$ & $A_{s}$ & $Z$ & \multicolumn{1}{l}{$A_{s}$} \\
\hline 0.184 & 1.65 & 0.090 & 3.88 \\
0.180 & 1.85 & 0.080 & 4.25 \\
0.170 & 2.06 & 0.070 & 4.70 \\
0.160 & 2.26 & 0.060 & 5.26 \\
0.150 & 2.44 & 0.050 & 5.98 \\
0.140 & 2.62 & 0.040 & 6.98 \\
0.130 & 2.82 & 0.035 & 7.62 \\
0.120 & 3.05 & 0.030 & 8.42 \\
0.110 & 3.29 & 0.026 & 9.24 \\
0.100 & 3.56 & 0.023 & 10.0 \\
\hline
\end{tabular}

\section{Relating theory to design}

The constant $Z$ is a useful figure to describe the effectiveness of a specific operational amplifier in meeting the specifications of a given design problem. It can be noted from eqn. 9 that $Z$ depends not only on the operational 
amplifier $G B W$, but also on the required overall gain and bandwidth of the amplifier to be designed. Eqn. 8 can be used to obtain the graph of Fig. 3 showing $Z$ as a function

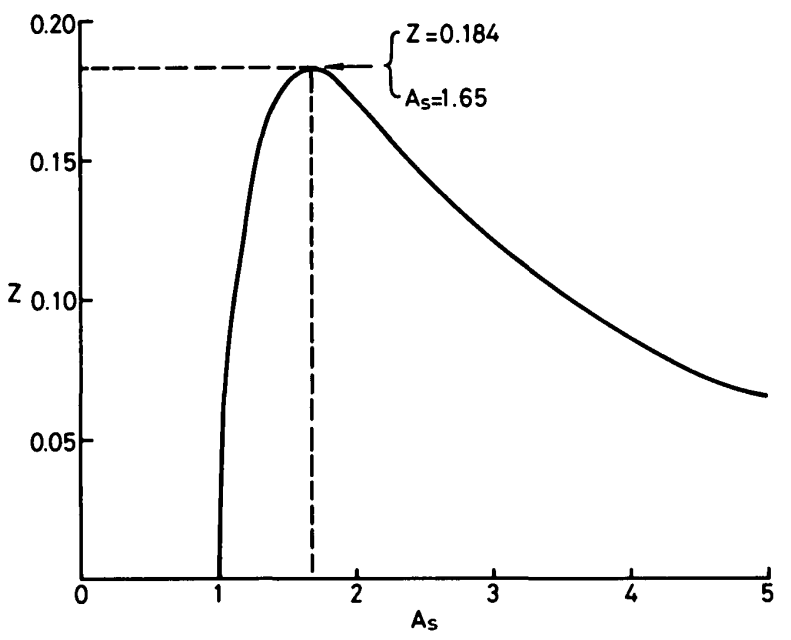

Fig. 3 Required value of $Z$ as a function of single stage gain

of $A_{s}$. The maximum value of $Z$ occurs when $A_{s}=1.65$. This value is 0.184 . For any value of gain, $Z$ must be less than or equal to the value 0.184 . If an operational amplifier is selected that leads to a value of $Z$ from eqn. 9 that exceeds 0.184 , it is immediately known that the operational amplifier cannot satisfy the overall amplifier specifications. If $Z$ equals 0.184 , then the interstage gain must equal 1.65 to achieve the desired overall gain and bandwidth. If $Z$ is less than 0.184 , the number of stages can be minimised as outlined in the previous section. The designer can immediately evaluate the suitability of a particular operational amplifier for the specified amplifier simply by calculating $Z$. The operational amplifier leading to the lowest value of $Z$ will result in the least number of stages required, assuming $Z$ is less than 0.184 . Although $Z$ can be considered to be a figure of merit, it is to be emphasised that lower values of $Z$ are most desirable.

As the cost of an operational amplifier is determined by production volume and performance of the operational amplifier in addition to other factors, there is no analytic method of minimising the overall amplifier cost. The minimum number of stages calculated for one operational amplifier will often be different from that calculated for a second operational amplifier with a different $G B W$. For example, operational amplifier A may require six stages, whereas operational amplifier B which is $20 \%$ more expensive than operational amplifier $A$, may require only four stages to satisfy the amplifier specifications. Thus, component cost minimisation will generally require trial-anderror methods.

The design procedure consists of the following steps:

(a) calculate the figure of merit $Z$ for the operational amplifier

(b) find the values of single stage gain $A_{s}$ from Table 1 or by solving eqn. 8

(c) calculate $m$, the minimum number of stages required, from eqn. 10

(d) repeat for all other operational amplifiers under consideration

(e) compare component cost for each case.

\section{A design example}

An amplifier is to be designed with an overall gain of 800 and an overall bandwidth of $100 \mathrm{kHz}$. The maximum output signal will be small enough to neglect slew rate limiting. Three types of operational amplifier are available, as shown in Table 2.

Table 2: Available types of operational amplifier

\begin{tabular}{lll}
\hline Operational amplifier & GBW & cost \\
\hline A & $2 \times 10^{5}$ & $x$ \\
B & $1 \times 10^{6}$ & $1.23 x$ \\
C & $1.5 \times 10^{6}$ & $1.95 x$ \\
\hline
\end{tabular}

The first step in the procedure calls for $Z$ to be calculated. For operational amplifier $A$, the figure of merit is found to be $Z=2.4$ which exceeds the maximum allowable value of 0.184 ; hence, this operational amplifier cannot be used. For operational amplifier $\mathrm{B}, Z=0.096$. The value of $A_{s}$ corresponding to this value of $Z$ is found by interpolation from Table 1 to be $A_{s}=3.68$. Applying eqn. 10 results in a minimum number of stages of 5.13. For a practical amplifier, an integer number of stages is required. Six stages would be used in this case. Repeating the calculations for operational amplifier $C$ yields values of $Z=0.043, A_{s}=6.68$, and $m=3.52$. Four stages would be required if operational amplifier $\mathrm{C}$ is used for the amplifier. Before choosing the operational amplifier, the overall device cost is compared. For operational amplifier B, the device cost is $6(1.23 x)=7.38 x$ and the device cost for operational amplifier $C$ is $4(1.95 x)=7.80 x$. Although the amplifier requires fewer stages when using operational amplifier $\mathrm{C}$, the device cost for the overall amplifier is less when realised with operational amplifier $\mathrm{B}$.

The result obtained in this example should be compared to the number of stages required to optimise the overall bandwidth. In this case $A_{s}=1.65$ and $n=14$ stages. If classical theory is applied, 14 stages would be used rather than 4 operational amplifier $\mathrm{C}$ or 6 operational amplifier $\mathrm{B}$ stages.

In practice, a given operational amplifier type will exhibit broad variations of $G B W$ from one device to the next. The procedure outlined assumes equal values of $G B W$ for all devices. For practical amplifiers, the designer is generally interested in worst-case design. If several amplifiers are to be manufactured, each must meet minimum specifications even for that possible combination of stages that minimise performance. With the proposed method, minimum performance occurs when individual stage values of $G B W$ are lowest. Thus, if the value used to calculate $Z$ in eqn. 9 is $G B W_{\min }$ for the operational amplifier used, the resulting amplifier is the worst-case design.

\section{Conclusions}

The procedure discussed in the paper allows a designer to realise a specified overall gain and bandwidth of an amplifier while minimising the number of required stages. The figure of merit $Z$ is a useful parameter in determining whether or not a particular operational amplifier can be used in the specified amplifier design. This parameter also aids the designer in calculating the required gain per stage and the minimum number of required stages. Worst-case design can be carried out with this procedure and overall component cost can be minimised.

\section{References}

1 WATSON, J.: 'Analogue and switching circuit design' (Adam Hilger, Bristol, 1984), Chap. 7 
2 WAIT, J.V., HUELSMAN, L.P., and KORN, G.A.: 'Introduction to operational amplifier theory and applications' (McGraw-Hill, New York, 1975), Chap. 1

3 COMER, D.J.: 'Video amplifier design based on operational amplifiers', IEEE Trans., 1976, CAS-23, pp. 169-171

4 VALLEY, G.E., and WALLMAN, H.: 'Vacuum tube amplifiers' (McGraw-Hill MIT Radiation Lab Series, New York, 1948, Vol. 18), Chap. 4

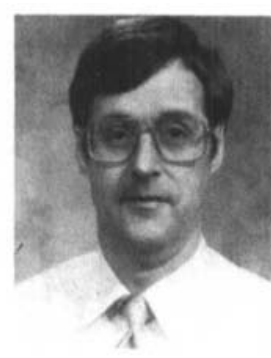

David J. Comer received his B.S. (1961), M.S. (1962), and Ph.D. (1966) degrees from San Jose State University, the University of California (Berkeley), and Washington State University, respectively, all in electrical engineering. Prom 1959 to 1964 he was employed by IBM Corporation in San Jose, California. In 1964, he taught part-time at San Jose State University. From 1964 to 1966 he was an Assistant Professor at the University of Idaho. From 1966 to 1969 he
5 GHAUSI, M.S. 'Electronic devices and circuits: discrete and integrated' (Holt, Rinehart and Winston, New York, 1985), Chap. 8

6 SEDRA, A.S., and SMITH, K.C.: 'Microelectronic circuits' (Holt, Rinehart and Winston, New York, 1982), Chap. 11

7 COMER, D.J.: 'Electronic design with integrated circuits' (AddisonWesley, Reading, 1981), Chap. 1

8 COMER, D.J., and GRIFFITH, J.M.: 'Optimisation of bandwidth in noniterative amplifiers', Proc. IEE, 1969, 116, (3), p. 384

was an Associate Professor at the University of Calgary in Calgary, Canada. In 1969, Prof. Comer became Professor and Chairman of the Division of Engineering at California State University in Chico, California. He joined the faculty at Brigham Young University in 1981 as a Professor. He holds the College of Engineering and Technology Research Chair for the 1984-85 academic year. Prof. Comer is the author of six textbooks and several articles in the areas of circuit design and computers. He holds two US patents and is a consultant in the area of digital systems. His fields of interest include microprocessor systems, electronic circuit design, and voice control of robots. 